\title{
CERTAIN CLASSES OF BI-UNIVALENT FUNCTIONS RELATED TO SHELL-LIKE CURVES CONNECTED WITH FIBONACCI NUMBERS
}

\author{
N. MAGESH, V. K. BALAJI AND C. ABIRAMI
}

\begin{abstract}
Recently, in their pioneering work on the subject of bi-univalent functions, Srivastava et al. 23] actually revived the study of the coefficient problems involving bi-univalent functions. Inspired by the pioneering work of Srivastava et al. [23], there has been triggering interest to study the coefficient problems for the different subclasses of bi-univalent functions. Motivated largely by Ali et al. [1, Srivastava et al. 23] and Güney et al. 10 in this paper, we consider certain classes of bi-univalent functions related to shell-like curves connected with Fibonacci numbers to obtain the estimates of second, third Taylor-Maclaurin coefficients and Fekete - Szegö inequalities. Further, certain special cases are also indicated. Some interesting remarks of the results presented here are also discussed.
\end{abstract}

\section{INTRODUCTION AND DEFINITIONS}

Let $\mathbb{R}=(-\infty, \infty)$ be the set of real numbers, $\mathbb{C}$ be the set of complex numbers and

$$
\mathbb{N}:=\{1,2,3, \ldots\}=\mathbb{N}_{0} \backslash\{0\}
$$

be the set of positive integers. Let $\mathcal{A}$ denote the class of functions of the form

$$
f(z)=z+\sum_{n=2}^{\infty} a_{n} z^{n}
$$

which are analytic in the open unit disk $\mathbb{D}=\{z: z \in \mathbb{C}$ and $|z|<1\}$. Further, by $\mathcal{S}$ we shall denote the class of all functions in $\mathcal{A}$ which are univalent in $\mathbb{D}$.

Let $\mathcal{P}$ denote the class of functions of the form

$$
p(z)=1+p_{1} z+p_{2} z^{2}+p_{3} z^{3}+\ldots \quad(z \in \mathbb{D})
$$

which are analytic with $\Re\{p(z)\}>0$. Here $p(z)$ is called as Caratheodory functions [8]. It is well known that the following correspondence between the class $\mathcal{P}$ and the class of Schwarz functions $w$ exists: $p \in \mathcal{P}$ if and only if $p(z)=1+w(z) / 1-w(z)$. Let $\mathcal{P}(\beta)$, $0 \leq \beta<1$, denote the class of analytic functions $p$ in $\mathbb{D}$ with $p(0)=1$ and $\Re\{p(z)\}>\beta$.

For analytic functions $f$ and $g$ in $\mathbb{D}, f$ is said to be subordinate to $g$ if there exists an analytic function $w$ such that

$$
w(0)=0, \quad|w(z)|<1 \quad \text { and } \quad f(z)=g(w(z)) \quad(z \in \mathbb{D}) .
$$

This subordination will be denoted here by

$$
f \prec g \quad(z \in \mathbb{D})
$$

or, conventionally, by

$$
f(z) \prec g(z) \quad(z \in \mathbb{D}) .
$$

2010 Mathematics Subject Classification. Primary 30C45; Secondary 30C50.

Key words and phrases. Univalent functions, bi-univalent functions, shell-like function, convex shelllike function, pseudo starlike function, Bazilević function. 
In particular, when $g$ is univalent in $\mathbb{D}$,

$$
f \prec g \quad(z \in \mathbb{D}) \Leftrightarrow f(0)=g(0) \quad \text { and } \quad f(\mathbb{D}) \subset g(\mathbb{D}) .
$$

Some of the important and well-investigated subclasses of the univalent function class $\mathcal{S}$ include (for example) the class $\mathcal{S}^{*}(\alpha)$ of starlike functions of order $\alpha(0 \leqq \alpha<1)$ in $\mathbb{D}$ and the class $\mathcal{K}(\alpha)$ of convex functions of order $\alpha(0 \leqq \alpha<1)$ in $\mathbb{D}$, the class $\mathcal{S}^{*}(\varphi)$ of Ma-Minda starlike functions and the class $\mathcal{K}(\varphi)$ of Ma-Minda convex functions $(\varphi$ is an analytic function with positive real part in $\mathbb{D}, \varphi(0)=1, \varphi^{\prime}(0)>0$ and $\varphi$ maps $\mathbb{D}$ onto a region starlike with respect to 1 and symmetric with respect to the real axis) (see [8]).

It is well known that every function $f \in \mathcal{S}$ has an inverse $f^{-1}$, defined by

$$
f^{-1}(f(z))=z \quad(z \in \mathbb{D})
$$

and

$$
f\left(f^{-1}(w)\right)=w \quad\left(|w|<r_{0}(f) ; r_{0}(f) \geqq \frac{1}{4}\right),
$$

where

$$
f^{-1}(w)=w-a_{2} w^{2}+\left(2 a_{2}^{2}-a_{3}\right) w^{3}-\left(5 a_{2}^{3}-5 a_{2} a_{3}+a_{4}\right) w^{4}+\ldots
$$

A function $f \in \mathcal{A}$ is said to be bi-univalent in $\mathbb{D}$ if both $f(z)$ and $f^{-1}(z)$ are univalent in $\mathbb{D}$. Let $\Sigma$ denote the class of bi-univalent functions in $\mathbb{D}$ given by (1.1). Recently, in their pioneering work on the subject of bi-univalent functions, Srivastava et al. [23] actually revived the study of the coefficient problems involving bi-univalent functions. Various subclasses of the bi-univalent function class $\Sigma$ were introduced and non-sharp estimates on the first two coefficients $\left|a_{2}\right|$ and $\left|a_{3}\right|$ in the Taylor-Maclaurin series expansion (1.1) were found in several recent investigations (see, for example, [1, 2, 3, 4, 15, 9, 11, 17, 18, $19,20,21,22,24,25,26]$ and references therein). The afore-cited all these papers on the subject were actually motivated by the pioneering work of Srivastava et al. [23]. However, the problem to find the coefficient bounds on $\left|a_{n}\right|(n=3,4, \ldots)$ for functions $f \in \Sigma$ is still an open problem.

The classes $\mathcal{S} \mathcal{L}(\tilde{p})$ and $\mathcal{K} \mathcal{S} \mathcal{L}(\tilde{p})$ of shell-like functions and convex shell-like functions are respectively, characterized by $z f^{\prime} / f(z) \prec \tilde{p}(z)$ or $1+z^{2} f^{\prime \prime} / f^{\prime}(z) \prec \tilde{p}(z)$, where $\tilde{p}(z)=$ $\left(1+\tau^{2} z^{2}\right) /\left(1-\tau z-\tau^{2} z^{2}\right), \tau=(1-\sqrt{5}) / 2 \approx-0.618$. The classes $\mathcal{S} \mathcal{L}(\tilde{p})$ and $\mathcal{K} \mathcal{S} \mathcal{L}(\tilde{p})$ were introduced and studied by Sokół [16] and Dziok et al. [6] respectively (see also [7, 15]). The function $\tilde{p}$ is not univalent in $\mathbb{D}$, but it is univalent in the disc $|z|<(3-\sqrt{5}) / 2 \approx$ 0.38. For example, $\tilde{p}(0)=\tilde{p}(-1 / 2 \tau)=1$ and $\tilde{p}\left(e^{\mp} \arccos (1 / 4)\right)=\sqrt{5} / 5$ and it may also be noticed that $1 /|\tau|=|\tau| / 1-|\tau|$ which shows that the number $|\tau|$ divides $[0,1]$ such that it fulfills the golden section. The image of the unit circle $|z|=1$ under $\tilde{p}$ is a curve described by the equation given by $(10 x-\sqrt{5}) y^{2}=(\sqrt{5}-2 x)(\sqrt{5} x-1)^{2}$, which is translated and revolved trisectrix of Maclaurin. The curve $\tilde{p}\left(r e^{i t}\right)$ is a closed curve without any loops for $0<r \leq r_{0}=(3-\sqrt{5}) / 2 \approx 0.38$. For $r_{0}<r<1$, it has a loop and for $r=1$, it has a vertical asymptote. Since $\tau$ satisfies the equation $\tau^{2}=1+\tau$, this expression can be used to obtain higher powers $\tau^{n}$ as a linear function of lower powers, which in turn can be decomposed all the way down to a linear combination of $\tau$ and 1 . The resulting recurrence relationships yield Fibonacci numbers $u_{n}$

$$
\tau^{n}=u_{n} \tau+u_{n-1} .
$$


Recently Raina and Sokół [15], taking $\tau z=t$, showed that

$$
\tilde{p}(z)=\frac{1+\tau^{2} z^{2}}{1-\tau z-\tau^{2} z^{2}}=1+\sum_{n=2}^{\infty}\left(u_{n-1}+u_{n+1}\right) \tau^{n} z^{n},
$$

where

$$
u_{n}=\frac{(1-\tau)^{n}-\tau}{\sqrt{5}}, \quad \tau=\frac{1-\sqrt{5}}{2}, ; \quad n=1,2, \ldots
$$

This shows that the relevant connection of $\tilde{p}$ with the sequence of Fibonacci numbers $u_{n}$, such that

$$
u_{0}=0, \quad u_{1}=1, \quad u_{n+2}=u_{n}+u_{n+1}
$$

for $n=0,1,2,3, \ldots$. Hence

$$
\tilde{p}(z)=1+\tau z+3 \tau^{2} z^{2}+4 \tau^{3} z^{3}+7 \tau^{4} z^{4}+11 \tau^{5} z^{5}+\ldots
$$

We note that the function $\tilde{p}$ belongs to the class $P(\beta)$ with $\beta=\frac{\sqrt{5}}{10} \approx 0.2236$ (see [15]).

Motivated by works of Ali et al. [1], Güney et al. [10] and Orhan et al. [13], we introduce the following new subclasses of bi-univalent functions, as follows.

Definition 1.1. A function $f \in \Sigma$ of the form

$$
f(z)=z+\sum_{n=2}^{\infty} a_{n} z^{n}
$$

belongs to the class $\mathcal{W} \mathcal{S}_{\Sigma}(\gamma, \lambda, \alpha, \tilde{p}), \gamma \in \mathbb{C} \backslash\{0\}, \alpha \geq 0$ and $\lambda \geq 0$, if the following conditions are satisfied:

$1+\frac{1}{\gamma}\left((1-\alpha+2 \lambda) \frac{f(z)}{z}+(\alpha-2 \lambda) f^{\prime}(z)+\lambda z f^{\prime \prime}(z)-1\right) \prec \widetilde{p(z)}=\frac{1+\tau^{2} z^{2}}{1-\tau z-\tau^{2} z^{2}}, z \in \mathbb{D}$

and for $g(w)=f^{-1}(w)$

$1+\frac{1}{\gamma}\left((1-\alpha+2 \lambda) \frac{g(w)}{w}+(\alpha-2 \lambda) g^{\prime}(w)+\lambda w g^{\prime \prime}(w)-1\right) \prec \widetilde{p(w)}=\frac{1+\tau^{2} w^{2}}{1-\tau w-\tau^{2} w^{2}}, w \in \mathbb{D}$,

where $\tau=\frac{1-\sqrt{5}}{2} \approx-0.618$

It is interesting to note that the special values of $\alpha, \gamma$ and $\lambda$ lead the class $\mathcal{W} \mathcal{S} \mathcal{L}_{\Sigma}(\gamma, \lambda, \alpha, \tilde{p})$ to various subclasses, we illustrate the following subclasses:

(1) For $\alpha=1+2 \lambda$, we get the class $\mathcal{W} \mathcal{S}_{\Sigma}(\gamma, \lambda, 1+2 \lambda, \tilde{p}) \equiv \mathcal{F} \mathcal{S} \mathcal{L}_{\Sigma}(\gamma, \lambda, \tilde{p})$. A function $f \in \Sigma$ of the form

$$
f(z)=z+\sum_{n=2}^{\infty} a_{n} z^{n}
$$

is said to be in $\mathcal{F} \mathcal{S} \mathcal{L}_{\Sigma}(\gamma, \lambda, \tilde{p})$, if the following conditions

$$
1+\frac{1}{\gamma}\left(f^{\prime}(z)+\lambda z f^{\prime \prime}(z)-1\right) \prec \widetilde{p(z)}=\frac{1+\tau^{2} z^{2}}{1-\tau z-\tau^{2} z^{2}}, z \in \mathbb{D}
$$


and for $g(w)=f^{-1}(w)$

$$
1+\frac{1}{\gamma}\left(g^{\prime}(w)+\lambda w g^{\prime \prime}(w)-1\right) \prec \widetilde{p(w)}=\frac{1+\tau^{2} w^{2}}{1-\tau w-\tau^{2} w^{2}}, w \in \mathbb{D},
$$

hold, where $\tau=\frac{1-\sqrt{5}}{2} \approx-0.618$.

(2) For $\lambda=0$, we obtain the class $\mathcal{W} \mathcal{S L}_{\Sigma}(\gamma, 0, \alpha, \tilde{p}) \equiv \mathcal{B S} \mathcal{L}_{\Sigma}(\gamma, \alpha, \tilde{p})$. A function $f \in \Sigma$ of the form

$$
f(z)=z+\sum_{n=2}^{\infty} a_{n} z^{n}
$$

is said to be in $\mathcal{B S}_{\mathcal{L}}(\gamma, \alpha, \tilde{p})$, if the following conditions

$$
1+\frac{1}{\gamma}\left((1-\alpha) \frac{f(z)}{z}+\alpha f^{\prime}(z)-1\right) \prec \widetilde{p(z)}=\frac{1+\tau^{2} z^{2}}{1-\tau z-\tau^{2} z^{2}}, z \in \mathbb{D}
$$

and for $g(w)=f^{-1}(w)$

$$
1+\frac{1}{\gamma}\left((1-\alpha) \frac{g(w)}{w}+\alpha g^{\prime}(w)-1\right) \prec \widetilde{p(w)}=\frac{1+\tau^{2} w^{2}}{1-\tau w-\tau^{2} w^{2}}, w \in \mathbb{D}
$$

hold, where $\tau=\frac{1-\sqrt{5}}{2} \approx-0.618$.

(3) For $\lambda=0$ and $\alpha=1$, we have the class $\mathcal{W} \mathcal{S}_{\Sigma}(\gamma, 0,1, \tilde{p}) \equiv \mathcal{H} \mathcal{S} \mathcal{L}_{\Sigma}(\gamma, \tilde{p})$. A function $f \in \Sigma$ of the form

$$
f(z)=z+\sum_{n=2}^{\infty} a_{n} z^{n}
$$

is said to be in $\mathcal{H} \mathcal{S} \mathcal{L}_{\Sigma}(\gamma, \tilde{p})$, if the following conditions

$$
1+\frac{1}{\gamma}\left(f^{\prime}(z)-1\right) \prec \widetilde{p(z)}=\frac{1+\tau^{2} z^{2}}{1-\tau z-\tau^{2} z^{2}}, z \in \mathbb{D}
$$

and for $g(w)=f^{-1}(w)$

$$
1+\frac{1}{\gamma}\left(g^{\prime}(w)-1\right) \prec \widetilde{p(w)}=\frac{1+\tau^{2} w^{2}}{1-\tau w-\tau^{2} w^{2}}, w \in \mathbb{D}
$$

hold, where $\tau=\frac{1-\sqrt{5}}{2} \approx-0.618$.

Definition 1.2. A function $f \in \Sigma$ of the form

$$
f(z)=z+\sum_{n=2}^{\infty} a_{n} z^{n}
$$

belongs to the class $\mathcal{R} \mathcal{S} \mathcal{L}_{\Sigma}(\gamma, \lambda, \tilde{p}), \gamma \in \mathbb{C} \backslash\{0\}$ and $\lambda \geq 0$, if the following conditions are satisfied:

$$
1+\frac{1}{\gamma}\left(\frac{z^{1-\lambda} f^{\prime}(z)}{(f(z))^{1-\lambda}}-1\right) \prec \widetilde{p(z)}=\frac{1+\tau^{2} z^{2}}{1-\tau z-\tau^{2} z^{2}}, z \in \mathbb{D}
$$

and for $g(w)=f^{-1}(w)$

$$
1+\frac{1}{\gamma}\left(\frac{w^{1-\lambda} g^{\prime}(w)}{(g(w))^{1-\lambda}}-1\right) \prec \widetilde{p(w)}=\frac{1+\tau^{2} w^{2}}{1-\tau w-\tau^{2} w^{2}}, w \in \mathbb{D},
$$


where $\tau=\frac{1-\sqrt{5}}{2} \approx-0.618$.

(1) For $\lambda=0$, we have the class $\mathcal{R} \mathcal{S}_{\Sigma}(\gamma, 0, \tilde{p}) \equiv \mathcal{S} \mathcal{L}_{\Sigma}(\gamma, \tilde{p})$. A function $f \in \Sigma$ of the form

$$
f(z)=z+\sum_{n=2}^{\infty} a_{n} z^{n}
$$

is said to be in $\mathcal{S L}_{\Sigma}(\gamma, \tilde{p})$, if the following conditions

$$
1+\frac{1}{\gamma}\left(\frac{z f^{\prime}(z)}{f(z)}-1\right) \prec \widetilde{p(z)}=\frac{1+\tau^{2} z^{2}}{1-\tau z-\tau^{2} z^{2}}, z \in \mathbb{D}
$$

and for $g(w)=f^{-1}(w)$

$$
1+\frac{1}{\gamma}\left(\frac{w g^{\prime}(w)}{g(w)}-1\right) \prec \widetilde{p(w)}=\frac{1+\tau^{2} w^{2}}{1-\tau w-\tau^{2} w^{2}}, w \in \mathbb{D}
$$

hold, where $\tau=\frac{1-\sqrt{5}}{2} \approx-0.618$.

Remark 1.3. For $\gamma=1$ the class $\mathcal{S} \mathcal{L}_{\Sigma}(1, \tilde{p}) \equiv \mathcal{S} \mathcal{L}_{\Sigma}(\tilde{p})$ was introduced and studied Güney et al. [10].

(2) For $\lambda=1$, we have the class $\mathcal{R} \mathcal{S} \mathcal{L}_{\Sigma}(\gamma, 1, \tilde{p}) \equiv \mathcal{H} \mathcal{S} \mathcal{L}_{\Sigma}(\gamma, \tilde{p})$.

Definition 1.4. A function $f \in \Sigma$ of the form

$$
f(z)=z+\sum_{n=2}^{\infty} a_{n} z^{n}
$$

belongs to the class $\mathcal{S L B}_{\Sigma}(\lambda ; \tilde{p}), \lambda \geq 1$, if the following conditions are satisfied:

$$
\frac{z\left[f^{\prime}(z)\right]^{\lambda}}{f(z)} \prec \widetilde{p(z)}=\frac{1+\tau^{2} z^{2}}{1-\tau z-\tau^{2} z^{2}}, z \in \mathbb{D}
$$

and for $g(w)=f^{-1}(w)$

$$
\frac{w\left[g^{\prime}(w)\right]^{\lambda}}{g(w)} \prec \widetilde{p(w)}=\frac{1+\tau^{2} w^{2}}{1-\tau w-\tau^{2} w^{2}}, w \in \mathbb{D},
$$

where $\tau=\frac{1-\sqrt{5}}{2} \approx-0.618$.

(1) For $\lambda=1$, we have the class $\mathcal{S} \mathcal{L} \mathcal{B}_{\Sigma}(1 ; \tilde{p}) \equiv \mathcal{S L}_{\Sigma}(\tilde{p})$. A function $f \in \Sigma$ of the form

$$
f(z)=z+\sum_{n=2}^{\infty} a_{n} z^{n}
$$

is said to be in $\mathcal{S} \mathcal{L}_{\Sigma}(\tilde{p})$, if the following conditions

$$
\frac{z f^{\prime}(z)}{f(z)} \prec \widetilde{p(z)}=\frac{1+\tau^{2} z^{2}}{1-\tau z-\tau^{2} z^{2}}, z \in \mathbb{D}
$$

and for $g(w)=f^{-1}(w)$

$$
\frac{w g^{\prime}(w)}{g(w)} \prec \widetilde{p(w)}=\frac{1+\tau^{2} w^{2}}{1-\tau w-\tau^{2} w^{2}}, w \in \mathbb{D},
$$


hold, where $\tau=\frac{1-\sqrt{5}}{2} \approx-0.618$.

Definition 1.5. A function $f \in \Sigma$ of the form

$$
f(z)=z+\sum_{n=2}^{\infty} a_{n} z^{n}
$$

belongs to the class $\mathcal{P} \mathcal{S} \mathcal{L}_{\Sigma}(\lambda ; \tilde{p}), 0 \leq \lambda \leq 1$, if the following conditions are satisfied:

$$
\frac{z f^{\prime}(z)+\lambda z^{2} f^{\prime \prime}(z)}{(1-\lambda) f(z)+\lambda z f^{\prime}(z)} \prec \widetilde{p(z)}=\frac{1+\tau^{2} z^{2}}{1-\tau z-\tau^{2} z^{2}}, z \in \mathbb{D}
$$

and for $g(w)=f^{-1}(w)$

$$
\frac{w f^{\prime}(w)+\lambda w^{2} g^{\prime \prime}(w)}{(1-\lambda) g(w)+\lambda w g^{\prime}(w)} \prec \widetilde{p(w)}=\frac{1+\tau^{2} w^{2}}{1-\tau w-\tau^{2} w^{2}}, w \in \mathbb{D},
$$

where $\tau=\frac{1-\sqrt{5}}{2} \approx-0.618$.

(1) For $\lambda=0$, we have the class $\mathcal{P} \mathcal{S} \mathcal{L}_{\Sigma}(0 ; \tilde{p}) \equiv \mathcal{S} \mathcal{L}_{\Sigma}(\tilde{p})$. A function $f \in \Sigma$ of the form

$$
f(z)=z+\sum_{n=2}^{\infty} a_{n} z^{n}
$$

is said to be in $\mathcal{S} \mathcal{L}_{\Sigma}(\tilde{p})$, if the following conditions

$$
\frac{z f^{\prime}(z)}{f(z)} \prec \widetilde{p(z)}=\frac{1+\tau^{2} z^{2}}{1-\tau z-\tau^{2} z^{2}}, z \in \mathbb{D}
$$

and for $g(w)=f^{-1}(w)$

$$
\frac{w g^{\prime}(w)}{g(w)} \prec \widetilde{p(w)}=\frac{1+\tau^{2} w^{2}}{1-\tau w-\tau^{2} w^{2}}, w \in \mathbb{D},
$$

hold, where $\tau=\frac{1-\sqrt{5}}{2} \approx-0.618$.

(2) For $\lambda=1$, we have the class $\mathcal{P} \mathcal{S L}_{\Sigma}(1 ; \tilde{p}) \equiv \mathcal{K} \mathcal{S} \mathcal{L}_{\Sigma}(\tilde{p})$. A function $f \in \Sigma$ of the form

$$
f(z)=z+\sum_{n=2}^{\infty} a_{n} z^{n}
$$

is said to be in $\mathcal{K} \mathcal{S} \mathcal{L}_{\Sigma}(\tilde{p})$, if the following conditions

$$
1+\frac{z^{2} f^{\prime \prime}(z)}{f^{\prime}(z)} \prec \widetilde{p(z)}=\frac{1+\tau^{2} z^{2}}{1-\tau z-\tau^{2} z^{2}}, z \in \mathbb{D}
$$

and for $g(w)=f^{-1}(w)$

$$
1+\frac{w^{2} g^{\prime \prime}(w)}{g^{\prime}(w)} \prec \widetilde{p(w)}=\frac{1+\tau^{2} w^{2}}{1-\tau w-\tau^{2} w^{2}}, w \in \mathbb{D},
$$

hold, where $\tau=\frac{1-\sqrt{5}}{2} \approx-0.618$.

Remark 1.6. For $\gamma=0, \mathcal{P} \mathcal{S} \mathcal{L}_{\Sigma}(0, \tilde{p}) \equiv \mathcal{S} \mathcal{L}_{\Sigma}(\tilde{p})$ and $\gamma=1, \mathcal{P} \mathcal{L}_{\Sigma}(1, \tilde{p}) \equiv \mathcal{K} \mathcal{S L}_{\Sigma}(\tilde{p})$ the classes were introduced and studied Güney et al. [10]. 
In order to prove our results for the function in the classes $\mathcal{W} \mathcal{S} \mathcal{L}_{\Sigma}(\gamma, \lambda, \alpha, \tilde{p}), \mathcal{R S}_{\mathcal{L}}(\gamma, \lambda, \tilde{p})$, $\mathcal{S} \mathcal{L} \mathcal{B}_{\Sigma}(\lambda ; \tilde{p})$ and $\mathcal{P} \mathcal{S} \mathcal{L}_{\Sigma}(\lambda ; \tilde{p})$, we need the following lemma.

Lemma 1.7. 14] If $p \in \mathcal{P}$, then $\left|p_{i}\right| \leqq 2$ for each $i$, where $\mathcal{P}$ is the family of all functions $p$, analytic in $\mathbb{D}$, for which

$$
\Re\{p(z)\}>0 \quad(z \in \mathbb{D}),
$$

where

$$
p(z)=1+p_{1} z+p_{2} z^{2}+\cdots \quad(z \in \mathbb{D}) .
$$

In this investigation, we find the estimates for the coefficients $\left|a_{2}\right|$ and $\left|a_{3}\right|$ for functions in the subclass $\mathcal{W} \mathcal{S} \mathcal{L}_{\Sigma}(\gamma, \lambda, \alpha, \tilde{p}), \mathcal{R} \mathcal{S} \mathcal{L}_{\Sigma}(\gamma, \lambda, \tilde{p}), \mathcal{S} \mathcal{L} \mathcal{B}_{\Sigma}(\lambda ; \tilde{p})$ and $\mathcal{P} \mathcal{S} \mathcal{L}_{\Sigma}(\lambda ; \tilde{p})$ Also, we obtain the upper bounds using the results of $\left|a_{2}\right|$ and $\left|a_{3}\right|$.

\section{Initial Coefficient Estimates and Fekete-Szegö Inequalities}

In the following theorem, we obtain coefficient estimates for functions in the class $f \in \mathcal{W} \mathcal{S} \mathcal{L}_{\Sigma}(\gamma, \lambda, \alpha, \tilde{p})$.

Theorem 2.1. Let $f(z)=z+\sum_{n=2}^{\infty} a_{n} z^{n}$ be in the class $\mathcal{W} \mathcal{S} \mathcal{L}_{\Sigma}(\gamma, \lambda, \alpha, \tilde{p})$. Then

$$
\begin{gathered}
\left|a_{2}\right| \leq \frac{|\gamma||\tau|}{\sqrt{\gamma \tau(1+2 \alpha+2 \lambda)+(1-3 \tau)(1+\alpha)^{2}}} \\
\left|a_{3}\right| \leq \frac{|\gamma||\tau|\left\{(1-3 \tau)(1+\alpha)^{2}\right\}}{(1+2 \alpha+2 \lambda)\left[\gamma \tau(1+2 \alpha+2 \lambda)+(1-3 \tau)(1+\alpha)^{2}\right]}
\end{gathered}
$$

and

$$
\left|a_{3}-\mu a_{2}^{2}\right| \leq \begin{cases}\frac{\gamma|\tau|}{(1+2 \alpha+2 \lambda)} & ; 0 \leq|h(\mu)| \leq \frac{\gamma|\tau|}{4(1+2 \alpha+2 \lambda)} \\ 4|h(\mu)| & ;|h(\mu)| \geq \frac{\gamma|\tau|}{4(1+2 \alpha+2 \lambda)}\end{cases}
$$

where

$$
h(\mu)=\frac{(1-\mu) \gamma^{2} \tau^{2}}{4\left[\gamma \tau(1+2 \alpha+2 \lambda)+(1+\alpha)^{2}(1-3 \tau)\right]} .
$$

Proof. Since $f \in \mathcal{W} \mathcal{S} \mathcal{L}_{\Sigma}(\gamma, \lambda, \alpha, \tilde{p})$, from the Definition [1.1 we have

$$
1+\frac{1}{\gamma}\left((1-\alpha+2 \lambda) \frac{f(z)}{z}+(\alpha-2 \lambda) f^{\prime}(z)+\lambda z f^{\prime \prime}(z)-1\right)=\widetilde{p(u(z))}
$$

and

$$
1+\frac{1}{\gamma}\left((1-\alpha+2 \lambda) \frac{g(w)}{w}+(\alpha-2 \lambda) g^{\prime}(w)+\lambda w g^{\prime \prime}(w)-1\right)=\widetilde{p(v(w))},
$$

where $z, w \in \mathbb{D}$ and $g=f^{-1}$. Using the fact the function $p(z)$ of the form

$$
p(z)=1+p_{1} z+p_{2} z^{2}+\ldots
$$

and $p \prec \tilde{p}$. Then there exists an analytic function $u$ such that $|u(z)|<1$ in $\mathbb{D}$ and $p(z)=\tilde{p}(u(z))$. Therefore, define the function

$$
h(z)=\frac{1+u(z)}{1-u(z)}=1+c_{1} z+c_{2} z^{2}+\ldots
$$


is in the class $\mathcal{P}$. It follows that

$$
u(z)=\frac{h(z)-1}{h(z)+1}=\frac{c_{1}}{2} z+\left(c_{2}-\frac{c_{1}^{2}}{2}\right) \frac{z^{2}}{2}+\left(c_{3}-c_{1} c_{2}+\frac{c_{1}^{3}}{4}\right) \frac{z^{3}}{2}+\ldots
$$

and

$$
\begin{aligned}
\tilde{p}(u(z))=1+ & \tilde{p}\left(\frac{c_{1}}{2} z+\left(c_{2}-\frac{c_{1}^{2}}{2}\right) \frac{z^{2}}{2}+\left(c_{3}-c_{1} c_{2}+\frac{c_{1}^{3}}{4}\right) \frac{z^{3}}{2}+\ldots\right) \\
& +\tilde{p}_{2}\left(\frac{c_{1}}{2} z+\left(c_{2}-\frac{c_{1}^{2}}{2}\right) \frac{z^{2}}{2}+\left(c_{3}-c_{1} c_{2}+\frac{c_{1}^{3}}{4}\right) \frac{z^{3}}{2}+\ldots\right)^{2} \\
& +\tilde{p}_{3}\left(\frac{c_{1}}{2} z+\left(c_{2}-\frac{c_{1}^{2}}{2}\right) \frac{z^{2}}{2}+\left(c_{3}-c_{1} c_{2}+\frac{c_{1}^{3}}{4}\right) \frac{z^{3}}{2}+\ldots\right)^{3}+\ldots \\
=1 & +\frac{\tilde{p}_{1} c_{1}}{2} z+\left(\frac{1}{2}\left(c_{2}-\frac{c_{1}^{2}}{2}\right) \tilde{p}_{1}+\frac{c_{1}^{2}}{4} \tilde{p}_{2}\right) z^{2} \\
& +\left(\frac{1}{2}\left(c_{3}-c_{1} c_{2}+\frac{c_{1}^{3}}{4}\right) \tilde{p}_{1}+\frac{1}{2} c_{1}\left(c_{2}-\frac{c_{1}^{2}}{2}\right) \tilde{p}_{2}+\frac{c_{1}^{3}}{8} \tilde{p}_{3}\right) z^{3}+\ldots
\end{aligned}
$$

Similarly, there exists an analytic function $v$ such that $|v(w)|<1$ in $\mathbb{D}$ and $p(w)=\tilde{p}(v(w))$. Therefore, the function

$$
k(w)=\frac{1+v(w)}{1-v(w)}=1+d_{1} w+d_{2} w^{2}+\ldots
$$

is in the class $\mathcal{P}$. It follows that

$$
v(w)=\frac{k(w)-1}{k(w)+1}=\frac{d_{1}}{2} w+\left(d_{2}-\frac{d_{1}^{2}}{2}\right) \frac{w^{2}}{2}+\left(d_{3}-d_{1} d_{2}+\frac{d_{1}^{3}}{4}\right) \frac{w^{3}}{2}+\ldots
$$

and

$$
\begin{aligned}
\tilde{p}(v(w))=1 & +\tilde{p}\left(\frac{d_{1}}{2} w+\left(d_{2}-\frac{d_{1}^{2}}{2}\right) \frac{w^{2}}{2}+\left(d_{3}-d_{1} d_{2}+\frac{d_{1}^{3}}{4}\right) \frac{w^{3}}{2}+\ldots\right) \\
& +\tilde{p}_{2}\left(\frac{d_{1}}{2} w+\left(d_{2}-\frac{d_{1}^{2}}{2}\right) \frac{w^{2}}{2}+\left(d_{3}-d_{1} d_{2}+\frac{d_{1}^{3}}{4}\right) \frac{w^{3}}{2}+\ldots\right)^{2} \\
& +\tilde{p}_{3}\left(\frac{d_{1}}{2} w+\left(d_{2}-\frac{d_{1}^{2}}{2}\right) \frac{w^{2}}{2}+\left(d_{3}-d_{1} d_{2}+\frac{d_{1}^{3}}{4}\right) \frac{w^{3}}{2}+\ldots\right)^{3}+\ldots \\
= & +\frac{\tilde{p}_{1} d_{1}}{2} w+\left(\frac{1}{2}\left(d_{2}-\frac{d_{1}^{2}}{2}\right) \tilde{p}_{1}+\frac{d_{1}^{2}}{4} \tilde{p}_{2}\right) w^{2} \\
& +\left(\frac{1}{2}\left(d_{3}-d_{1} d_{2}+\frac{d_{1}^{3}}{4}\right) \tilde{p}_{1}+\frac{1}{2} d_{1}\left(d_{2}-\frac{d_{1}^{2}}{2}\right) \tilde{p}_{2}+\frac{d_{1}^{3}}{8} \tilde{p}_{3}\right) w^{3} \\
& +\ldots
\end{aligned}
$$

By virtue of (2.1), (2.2), (2.3) and (2.4), we have

$$
\begin{gathered}
\frac{1}{\gamma}(1+\alpha) a_{2}=\frac{c_{1} \tau}{2}, \\
\frac{a_{3}}{\gamma}(1+2 \alpha+2 \lambda)=\frac{1}{2}\left(c_{2}-\frac{c_{1}^{2}}{2}\right) \tau+\frac{3 c_{1}^{2}}{4} \tau^{2},
\end{gathered}
$$




$$
-\frac{1}{\gamma}(1+\alpha) a_{2}=\frac{d_{1} \tau}{2}
$$

and

$$
\frac{(1+2 \alpha+2 \lambda)}{\gamma}\left(2 a_{2}^{2}-a_{3}\right)=\frac{1}{2}\left(d_{2}-\frac{d_{1}^{2}}{2}\right) \tau+\frac{3 d_{1}^{2}}{4} \tau^{2} .
$$

From (2.5) and (2.7), we obtain

$$
c_{1}=-d_{1}
$$

and

$$
\begin{aligned}
\frac{2}{\gamma^{2}}(1+\alpha)^{2} a_{2}^{2} & =\frac{\left(c_{1}^{2}+d_{1}^{2}\right) \tau^{2}}{4} \\
a_{2}^{2} & =\frac{\gamma^{2}\left(c_{1}^{2}+d_{1}^{2}\right) \tau^{2}}{8(1+\alpha)^{2}} .
\end{aligned}
$$

By adding (2.6) and (2.8), we have

$$
\frac{2}{\gamma}(1+2 \alpha+2 \lambda) a_{2}^{2}=\frac{1}{2}\left(c_{2}+d_{2}\right) \tau-\frac{1}{4}\left(c_{1}^{2}+d_{1}^{2}\right) \tau+\frac{3}{4}\left(c_{1}^{2}+d_{1}^{2}\right) \tau^{2} .
$$

By substituting (2.9) in (2.10), we reduce that

$$
a_{2}^{2}=\frac{\gamma^{2}\left(c_{2}+d_{2}\right) \tau^{2}}{4\left[\gamma \tau(1+2 \alpha+2 \lambda)+(1-3 \tau)(1+\alpha)^{2}\right]} .
$$

Now, applying Lemma 1.7, we obtain

$$
\left|a_{2}\right| \leq \frac{|\gamma||\tau|}{\sqrt{\gamma \tau(1+2 \alpha+2 \lambda)+(1-3 \tau)(1+\alpha)^{2}}} .
$$

By subtracting (2.8) from (2.6), we obtain

$$
a_{3}=\frac{\gamma\left(c_{2}-d_{2}\right) \tau}{4(1+2 \alpha+2 \lambda)}+a_{2}^{2}
$$

Hence by Lemma 1.7, we have

$$
\left|a_{3}\right| \leq \frac{|\gamma|\left(\left|c_{2}\right|+\left|d_{2}\right|\right)|\tau|}{4(1+2 \alpha+2 \lambda)}+\left|a_{2}\right|^{2} \leq \frac{|\gamma||\tau|}{(1+2 \alpha+2 \lambda)}+\left|a_{2}\right|^{2} .
$$

Then in view of (2.12), we obtain

$$
\left|a_{3}\right| \leq \frac{|\gamma||\tau|\left\{(1-3 \tau)(1+\alpha)^{2}\right\}}{(1+2 \alpha+2 \lambda)\left[\gamma \tau(1+2 \alpha+2 \lambda)+(1-3 \tau)(1+\alpha)^{2}\right]}
$$

From (2.13), we have

$$
a_{3}-\mu a_{2}^{2}=\frac{\gamma\left(c_{2}-d_{2}\right) \tau}{4(1+2 \alpha+2 \lambda)}+(1-\mu) a_{2}^{2} .
$$

By substituting (2.11) in (2.15), we have

$$
\begin{aligned}
a_{3}-\mu a_{2}^{2} & =\frac{\gamma\left(c_{2}-d_{2}\right) \tau}{4(1+2 \alpha+2 \lambda)}+(1-\mu)\left(\frac{\gamma^{2}\left(c_{2}+d_{2}\right) \tau^{2}}{4\left[\gamma \tau(1+2 \alpha+2 \lambda)+(1-3 \tau)(1+\alpha)^{2}\right]}\right) \\
& =\left(h(\mu)+\frac{\gamma|\tau|}{4(1+2 \alpha+2 \lambda)}\right) c_{2}+\left(h(\mu)-\frac{\gamma|\tau|}{4(1+2 \alpha+2 \lambda)}\right) d_{2},
\end{aligned}
$$


where

$$
h(\mu)=\frac{(1-\mu) \gamma^{2} \tau^{2}}{4\left[\gamma \tau(1+2 \alpha+2 \lambda)+(1+\alpha)^{2}(1-3 \tau)\right]} .
$$

Thus by taking modulus of (2.16), we conclude that

$$
\left|a_{3}-\mu a_{2}^{2}\right| \leq \begin{cases}\frac{\gamma|\tau|}{(1+2 \alpha+2 \lambda)} & ; 0 \leq|h(\mu)| \leq \frac{\gamma|\tau|}{4(1+2 \alpha+2 \lambda)} \\ 4|h(\mu)| & ;|h(\mu)| \geq \frac{\gamma|\tau|}{4(1+2 \alpha+2 \lambda)} .\end{cases}
$$

Theorem 2.2. Let $f(z)=z+\sum_{n=2}^{\infty} a_{n} z^{n}$ be in the class $\mathcal{R} \mathcal{S} \mathcal{L}_{\Sigma}(\gamma, \lambda, \tilde{p})$. Then

$$
\begin{gathered}
\left|a_{2}\right| \leq \frac{\sqrt{2}|\gamma||\tau|}{\sqrt{\gamma \tau(2+\lambda)(1+\lambda)+2(1-3 \tau)(1+\lambda)^{2}}} \\
\left|a_{3}\right| \leq \frac{|\gamma||\tau|\left\{\gamma \tau(2+\lambda)(1+\lambda)+2(1-3 \tau)(1+\lambda)^{2}-2(2+\lambda) \gamma \tau\right\}}{(2+\lambda)\left[\gamma \tau(2+\lambda)(1+\lambda)+2(1-3 \tau)(1+\lambda)^{2}\right]}
\end{gathered}
$$

and

$$
\left|a_{3}-\mu a_{2}^{2}\right| \leq \begin{cases}\frac{|\gamma||\tau|}{2+\lambda} & ; 0 \leq|\mu-1| \leq \frac{M}{2|\gamma||\tau|(2+\lambda)} \\ \frac{2|1-\mu| \gamma^{2} \tau^{2}}{M} & ;|\mu-1| \geq \frac{M}{2|\gamma||\tau|(2+\lambda)}\end{cases}
$$

where

$$
M=\gamma \tau(2+\lambda)(1+\lambda)+2(1+\lambda)^{2}(1-3 \tau) .
$$

Proof. Since $f \in \mathcal{R} \mathcal{S} \mathcal{L}_{\Sigma}(\gamma, \lambda, \tilde{p})$, from the Definition 1.2 we have

$$
1+\frac{1}{\gamma}\left(\frac{z^{1-\lambda} f^{\prime}(z)}{(f(z))^{1-\lambda}}-1\right)=\widetilde{p(u(z))}
$$

and

$$
1+\frac{1}{\gamma}\left(\frac{w^{1-\lambda} g^{\prime}(w)}{(g(w))^{1-\lambda}}-1\right)=\widetilde{p(v(w))} .
$$

By virtue of (2.17), (2.18), (2.3) and (2.4), we get

$$
\begin{gathered}
\frac{1}{\gamma}(1+\lambda) a_{2}=\frac{c_{1} \tau}{2} \\
\frac{1}{\gamma}(2+\lambda)\left[a_{3}+(\lambda-1) \frac{a_{2}^{2}}{2}\right]=\frac{1}{2}\left(c_{2}-\frac{c_{1}^{2}}{2}\right) \tau+\frac{3 c_{1}^{2}}{4} \tau^{2}, \\
-\frac{1}{\gamma}(1+\lambda) a_{2}=\frac{d_{1} \tau}{2}
\end{gathered}
$$

and

$$
\frac{1}{\gamma}(2+\lambda)\left[(3+\lambda) \frac{a_{2}^{2}}{2}-a_{3}\right]=\frac{1}{2}\left(d_{2}-\frac{d_{1}^{2}}{2}\right) \tau+\frac{3 d_{1}^{2}}{4} \tau^{2} .
$$

From (2.19) and (2.21), we obtain

$$
c_{1}=-d_{1},
$$


and

$$
\begin{aligned}
\frac{2}{\gamma^{2}}(1+\lambda)^{2} a_{2}^{2} & =\frac{\left(c_{1}^{2}+d_{1}^{2}\right) \tau^{2}}{4} \\
a_{2}^{2} & =\frac{\gamma^{2}\left(c_{1}^{2}+d_{1}^{2}\right) \tau^{2}}{8(1+\lambda)^{2}} .
\end{aligned}
$$

By adding (2.20) and (2.22), we have

$$
\frac{1}{\gamma}(2+\lambda)(1+\lambda) a_{2}^{2}=\frac{1}{2}\left(c_{2}+d_{2}\right) \tau-\frac{1}{4}\left(c_{1}^{2}+d_{1}^{2}\right) \tau+\frac{3}{4}\left(c_{1}^{2}+d_{1}^{2}\right) \tau^{2} .
$$

By substituting (2.23) in (2.24), we reduce that

$$
a_{2}^{2}=\frac{\gamma^{2}\left(c_{2}+d_{2}\right) \tau^{2}}{2\left[\gamma \tau(2+\lambda)(1+\lambda)+2(1-3 \tau)(1+\lambda)^{2}\right]}
$$

Now, applying Lemma 1.7, we obtain

$$
\left|a_{2}\right| \leq \frac{\sqrt{2}|\gamma||\tau|}{\sqrt{\gamma \tau(2+\lambda)(1+\lambda)+2(1-3 \tau)(1+\lambda)^{2}}}
$$

By subtracting (2.22) from (2.20), we obtain

$$
a_{3}=\frac{\gamma\left(c_{2}-d_{2}\right) \tau}{4(2+\lambda)}+a_{2}^{2}
$$

Hence by Lemma 1.7, we have

$$
\left|a_{3}\right| \leq \frac{|\gamma|\left(\left|c_{2}\right|+\left|d_{2}\right|\right)|\tau|}{4(2+\lambda)}+\left|a_{2}\right|^{2} \leq \frac{|\gamma||\tau|}{(2+\lambda)}+\left|a_{2}\right|^{2} .
$$

Then in view of (2.26), we obtain

$$
\left|a_{3}\right| \leq \frac{|\gamma||\tau|\left\{\gamma \tau(2+\lambda)(1+\lambda)+2(1-3 \tau)(1+\lambda)^{2}-2(2+\lambda) \gamma \tau\right\}}{(2+\lambda)\left[\gamma \tau(2+\lambda)(1+\lambda)+2(1-3 \tau)(1+\lambda)^{2}\right]} .
$$

From (2.27), we have

$$
a_{3}-\mu a_{2}^{2}=\frac{\gamma\left(c_{2}-d_{2}\right) \tau}{4(2+\lambda)}+(1-\mu) a_{2}^{2} .
$$

By substituting (2.25) in (2.29), we have

$$
\begin{aligned}
a_{3}-\mu a_{2}^{2} & =\frac{\gamma\left(c_{2}-d_{2}\right) \tau}{4(2+\lambda)}+(1-\mu)\left(\frac{\gamma^{2}\left(c_{2}+d_{2}\right) \tau^{2}}{2\left[\gamma \tau(2+\lambda)(1+\lambda)+2(1+\lambda)^{2}(1-3 \tau)\right]}\right) \\
& =\left(h(\mu)+\frac{|\gamma||\tau|}{4(2+\lambda)}\right) c_{2}+\left(h(\mu)-\frac{|\gamma||\tau|}{4(2+\lambda)}\right) d_{2}
\end{aligned}
$$

where

$$
h(\mu)=\frac{(1-\mu) \gamma^{2} \tau^{2}}{2\left[\gamma \tau(2+\lambda)(1+\lambda)+2(1+\lambda)^{2}(1-3 \tau)\right]} .
$$


Thus by taking modulus of (2.30), we conclude that

$$
\left|a_{3}-\mu a_{2}^{2}\right| \leq \begin{cases}\frac{\gamma|\tau|}{(2+\lambda)} & ; 0 \leq|h(\mu)| \leq \frac{\gamma|\tau|}{4(2+\lambda)} \\ 4|h(\mu)| & ;|h(\mu)| \geq \frac{\gamma|\tau|}{4(2+\lambda)}\end{cases}
$$

This gives desired inequality.

Theorem 2.3. Let $f(z)=z+\sum_{n=2}^{\infty} a_{n} z^{n}$ be in the class $\mathcal{S} \mathcal{L} \mathcal{B}_{\Sigma}(\lambda ; \tilde{p})$. Then

$$
\begin{gathered}
\left|a_{2}\right| \leq \frac{|\tau|}{\sqrt{(2 \lambda-1)[\tau(3-5 \lambda)+2 \lambda-1]}} \\
\left|a_{3}\right| \leq \frac{|\tau|\left[(2 \lambda-1)^{2}-2\left(5 \lambda^{2}-4 \lambda+1\right) \tau\right\}}{(2 \lambda-1)(3 \lambda-1)[(3-5 \lambda) \tau+2 \lambda-1]}
\end{gathered}
$$

and

$$
\left|a_{3}-\mu a_{2}^{2}\right| \leq \begin{cases}\frac{|\gamma||\tau|}{3 \lambda-1} & ; 0 \leq|\mu-1| \leq \frac{M}{|\tau|(3 \lambda-1)} \\ \frac{|1-\mu| \tau^{2}}{M} & ;|\mu-1| \geq \frac{M}{|\tau|(3 \lambda-1)}\end{cases}
$$

where

$$
M=(2 \lambda-1)[\tau(3-5 \lambda)+2 \lambda-1] .
$$

Proof. Since $f \in \mathcal{S L B}_{\Sigma}(\lambda ; \tilde{p})$, from the Definition 1.4 we have

$$
\frac{z\left[f^{\prime}(z)\right]^{\lambda}}{f(z)}=\widetilde{p(u(z))}
$$

and

$$
\frac{w\left[g^{\prime}(w)\right]^{\lambda}}{g(w)}=\widetilde{p(v(w))}
$$

By virtue of (2.31), (2.32), (2.3) and (2.4), we get

$$
\begin{aligned}
(2 \lambda-1) a_{2} & =\frac{c_{1} \tau}{2} \\
(3 \lambda-1) a_{3}+\left(2 \lambda^{2}-4 \lambda+1\right) a_{2}^{2} & =\frac{1}{2}\left(c_{2}-\frac{c_{1}^{2}}{2}\right) \tau+\frac{3 c_{1}^{2}}{4} \tau^{2} \\
-(2 \lambda-1) a_{2} & =\frac{d_{1} \tau}{2}
\end{aligned}
$$

and

$$
\left(2 \lambda^{2}+2 \lambda-1\right) a_{2}^{2}-(3 \lambda-1) a_{3}=\frac{1}{2}\left(d_{2}-\frac{d_{1}^{2}}{2}\right) \tau+\frac{3 d_{1}^{2}}{4} \tau^{2} .
$$

From (2.33) and (2.35), we obtain

$$
c_{1}=-d_{1}
$$


and

$$
\begin{aligned}
2(2 \lambda-1)^{2} a_{2}^{2} & =\frac{\left(c_{1}^{2}+d_{1}^{2}\right) \tau^{2}}{4} \\
a_{2}^{2} & =\frac{\left(c_{1}^{2}+d_{1}^{2}\right) \tau^{2}}{8(2 \lambda-1)^{2}} .
\end{aligned}
$$

By adding (2.34) and (2.36), we have

$$
2 \lambda(2 \lambda-1) a_{2}^{2}=\frac{1}{2}\left(c_{2}+d_{2}\right) \tau-\frac{1}{4}\left(c_{1}^{2}+d_{1}^{2}\right) \tau+\frac{3}{4}\left(c_{1}^{2}+d_{1}^{2}\right) \tau^{2} .
$$

By substituting (2.37) in (2.38), we reduce that

$$
a_{2}^{2}=\frac{\left(c_{2}+d_{2}\right) \tau^{2}}{4(2 \lambda-1)[\tau(3-5 \lambda)+2 \lambda-1]} .
$$

Now, applying Lemma 1.7, we obtain

$$
\left|a_{2}\right| \leq \frac{|\tau|}{\sqrt{(2 \lambda-1)[\tau(3-5 \lambda)+2 \lambda-1]}} .
$$

By subtracting (2.36) from (2.34), we obtain

$$
a_{3}=\frac{\left(c_{2}-d_{2}\right) \tau}{4(3 \lambda-1)}+a_{2}^{2}
$$

Hence by Lemma 1.7, we have

$$
\left|a_{3}\right| \leq \frac{\left(\left|c_{2}\right|+\left|d_{2}\right|\right)|\tau|}{4(3 \lambda-1)}+\left|a_{2}\right|^{2} \leq \frac{|\tau|}{3 \lambda-1}+\left|a_{2}\right|^{2} .
$$

Then in view of (2.40), we obtain

$$
\left|a_{3}\right| \leq \frac{|\tau|\left[(2 \lambda-1)^{2}-2\left(5 \lambda^{2}-4 \lambda+1\right) \tau\right\}}{(2 \lambda-1)(3 \lambda-1)[(3-5 \lambda) \tau+2 \lambda-1]}
$$

From (2.41), we have

$$
a_{3}-\mu a_{2}^{2}=\frac{\left(c_{2}-d_{2}\right) \tau}{4(3 \lambda-1)}+(1-\mu) a_{2}^{2} .
$$

By substituting (2.39) in (2.43), we have

$$
\begin{aligned}
a_{3}-\mu a_{2}^{2} & =\frac{\left(c_{2}-d_{2}\right) \tau}{4(3 \lambda-1)}+(1-\mu)\left(\frac{\left(c_{2}+d_{2}\right) \tau^{2}}{4(2 \lambda-1)[\tau(3-5 \lambda)+2 \lambda-1]}\right) \\
& =\left(h(\mu)+\frac{|\tau|}{4(3 \lambda-1)}\right) c_{2}+\left(h(\mu)-\frac{|\tau|}{4(3 \lambda-1)}\right) d_{2},
\end{aligned}
$$

where

$$
h(\mu)=\frac{(1-\mu) \tau^{2}}{4(2 \lambda-1)[\tau(3-5 \lambda)+2 \lambda-1]} .
$$

Thus by taking modulus of (2.44), we conclude that

$$
\left|a_{3}-\mu a_{2}^{2}\right| \leq \begin{cases}\frac{|\tau|}{(3 \lambda-1)} & ; 0 \leq|h(\mu)| \leq \frac{|\tau|}{4(3 \lambda-1)} \\ 4|h(\mu)| & ;|h(\mu)| \geq \frac{|\tau|}{4(3 \lambda-1)}\end{cases}
$$


This gives desired inequality.

Theorem 2.4. Let $f(z)=z+\sum_{n=2}^{\infty} a_{n} z^{n}$ be in the class $\mathcal{P S} \mathcal{L}_{\Sigma}(\lambda ; \tilde{p})$. Then

$$
\begin{gathered}
\left|a_{2}\right| \leq \frac{|\tau|}{\sqrt{(1+\lambda)^{2}-2 \tau\left(2 \lambda^{2}+2 \lambda+1\right)}}, \\
\left|a_{3}\right| \leq \frac{|\tau|(1-4 \tau)(1+\lambda)^{2}}{2(1+2 \lambda)\left[(1+\lambda)^{2}-2 \tau\left(2 \lambda^{2}+2 \lambda+1\right)\right]}
\end{gathered}
$$

and

$$
\left|a_{3}-\mu a_{2}^{2}\right| \leq \begin{cases}\frac{|\tau|}{2+4 \lambda} & ; 0 \leq|\mu-1| \leq \frac{M}{2|\tau|(1+2 \lambda)} \\ \frac{|1-\mu| \tau^{2}}{M} & ;|\mu-1| \geq \frac{M}{2|\tau|(1+2 \lambda)}\end{cases}
$$

where

$$
M=(1+\lambda)^{2}-2 \tau\left(2 \lambda^{2}+2 \lambda+1\right) .
$$

Proof. Since $f \in \mathcal{P} \mathcal{S} \mathcal{L}_{\Sigma}(\lambda ; \tilde{p})$, from the Definition 1.5 we have

$$
\frac{z f^{\prime}(z)+\lambda z^{2} f^{\prime \prime}(z)}{(1-\lambda) f(z)+\lambda z f^{\prime}(z)}=\widetilde{p(u(z))}
$$

and

$$
\frac{w f^{\prime}(w)+\lambda w^{2} g^{\prime \prime}(w)}{(1-\lambda) g(w)+\lambda w g^{\prime}(w)}=\widetilde{p(v(w))} .
$$

By virtue of (2.45), (2.46), (2.3) and (2.4), we get

$$
\begin{gathered}
(1+\lambda) a_{2}=\frac{c_{1} \tau}{2} \\
2(1+2 \lambda) a_{3}-(1+\lambda)^{2} a_{2}^{2}=\frac{1}{2}\left(c_{2}-\frac{c_{1}^{2}}{2}\right) \tau+\frac{3 c_{1}^{2}}{4} \tau^{2}, \\
-(1+\lambda) a_{2}=\frac{d_{1} \tau}{2}
\end{gathered}
$$

and

$$
-2(1+2 \lambda) a_{3}-\left(\lambda^{2}-6 \lambda-3\right) a_{2}^{2}=\frac{1}{2}\left(d_{2}-\frac{d_{1}^{2}}{2}\right) \tau+\frac{3 d_{1}^{2}}{4} \tau^{2} .
$$

From (2.47) and (2.49), we obtain

$$
c_{1}=-d_{1}
$$

and

$$
\begin{aligned}
2(1+\lambda)^{2} a_{2}^{2} & =\frac{\left(c_{1}^{2}+d_{1}^{2}\right) \tau^{2}}{4} \\
a_{2}^{2} & =\frac{\left(c_{1}^{2}+d_{1}^{2}\right) \tau^{2}}{8(1+\lambda)^{2}}
\end{aligned}
$$


By adding (2.48) and (2.50), we have

$$
2\left(1+2 \lambda-\lambda^{2}\right) a_{2}^{2}=\frac{1}{2}\left(c_{2}+d_{2}\right) \tau-\frac{1}{4}\left(c_{1}^{2}+d_{1}^{2}\right) \tau+\frac{3}{4}\left(c_{1}^{2}+d_{1}^{2}\right) \tau^{2} .
$$

By substituting (2.51) in (2.52), we reduce that

$$
a_{2}^{2}=\frac{\left(c_{2}+d_{2}\right) \tau^{2}}{4\left[(1+\lambda)^{2}-2 \tau\left(2 \lambda^{2}+2 \lambda+1\right)\right]} .
$$

Now, applying Lemma 1.7, we obtain

$$
\left|a_{2}\right| \leq \frac{|\tau|}{\sqrt{(1+\lambda)^{2}-2 \tau\left(2 \lambda^{2}+2 \lambda+1\right)}} .
$$

By subtracting (2.50) from (2.48), we obtain

$$
a_{3}=\frac{\left(c_{2}-d_{2}\right) \tau}{8(1+2 \lambda)}+a_{2}^{2}
$$

Hence by Lemma 1.7, we have

$$
\left|a_{3}\right| \leq \frac{\left(\left|c_{2}\right|+\left|d_{2}\right|\right)|\tau|}{8(1+2 \lambda)}+\left|a_{2}\right|^{2} \leq \frac{|\tau|}{2+4 \lambda}+\left|a_{2}\right|^{2}
$$

Then in view of (2.54), we obtain

$$
\left|a_{3}\right| \leq \frac{|\tau|(1-4 \tau)(1+\lambda)^{2}}{2(1+2 \lambda)\left[(1+\lambda)^{2}-2 \tau\left(2 \lambda^{2}+2 \lambda+1\right)\right]}
$$

From (2.55), we have

$$
a_{3}-\mu a_{2}^{2}=\frac{\left(c_{2}-d_{2}\right) \tau}{8(1+2 \lambda)}+(1-\mu) a_{2}^{2} .
$$

By substituting (2.53) in (2.56), we have

$$
\begin{aligned}
a_{3}-\mu a_{2}^{2} & =\frac{\left(c_{2}-d_{2}\right) \tau}{8(1+2 \lambda)}+(1-\mu)\left(\frac{\left(c_{2}+d_{2}\right) \tau^{2}}{4\left[(1+\lambda)^{2}-2 \tau\left(2 \lambda^{2}+2 \lambda+1\right)\right]}\right) \\
& =\left(h(\mu)+\frac{\tau}{8(1+2 \lambda)}\right) c_{2}+\left(h(\mu)-\frac{\tau}{8(1+2 \lambda)}\right) d_{2},
\end{aligned}
$$

where

$$
h(\mu)=\frac{(1-\mu) \tau^{2}}{4\left[(1+\lambda)^{2}-2 \tau\left(2 \lambda^{2}+2 \lambda+1\right)\right]} .
$$

Thus by taking modulus of (2.57), we conclude that

$$
\left|a_{3}-\mu a_{2}^{2}\right| \leq \begin{cases}\frac{|\tau|}{2(1+2 \lambda)} & ; 0 \leq|h(\mu)| \leq \frac{|\tau|}{8(1+2 \lambda)} \\ 4|h(\mu)| & ;|h(\mu)| \geq \frac{|\tau|}{8(1+2 \lambda)} .\end{cases}
$$

This gives desired inequality. 


\section{Corollaries and Consequences}

Corollary 3.1. Let $f(z)=z+\sum_{n=2}^{\infty} a_{n} z^{n}$ be in the class $\mathcal{F} \mathcal{S L}_{\Sigma}(\gamma, \lambda, \tilde{p})$. Then $\left|a_{2}\right| \leq \frac{|\gamma||\tau|}{\sqrt{3 \gamma \tau(1+2 \lambda)+4(1-3 \tau)(1+\lambda)^{2}}}, \quad\left|a_{3}\right| \leq \frac{4|\gamma||\tau|(1-3 \tau)(1+\alpha)^{2}}{3(1+2 \lambda)\left[3 \gamma \tau(1+2 \lambda)+4(1-3 \tau)(1+\lambda)^{2}\right]}$ and

$$
\left|a_{3}-\mu a_{2}^{2}\right| \leq \begin{cases}\frac{|\gamma||\tau|}{3+6 \lambda} & ; 0 \leq|h(\mu)| \leq \frac{|\gamma||\tau|}{12+24 \lambda} \\ 4|h(\mu)| & ;|h(\mu)| \geq \frac{|\gamma||\tau|}{12+24 \lambda}\end{cases}
$$

where

$$
h(\mu)=\frac{(1-\mu) \gamma^{2} \tau^{2}}{4\left[3 \gamma \tau(1+2 \lambda)+4(1-3 \tau)(1+\lambda)^{2}\right]} .
$$

Corollary 3.2. Let $f(z)=z+\sum_{n=2}^{\infty} a_{n} z^{n}$ be in the class $\mathcal{B S} \mathcal{L}_{\Sigma}(\gamma, \alpha, \tilde{p})$. Then $\left|a_{2}\right| \leq \frac{|\gamma||\tau|}{\sqrt{\gamma \tau(1+2 \alpha)+(1-3 \tau)(1+\alpha)^{2}}}, \quad\left|a_{3}\right| \leq \frac{|\gamma||\tau|\left\{(1-3 \tau)(1+\alpha)^{2}\right\}}{(1+2 \alpha)\left[\gamma \tau(1+2 \alpha)+(1-3 \tau)(1+\alpha)^{2}\right]}$ and

$$
\left|a_{3}-\mu a_{2}^{2}\right| \leq \begin{cases}\frac{|\gamma||\tau|}{1+2 \alpha} & ; 0 \leq|h(\mu)| \leq \frac{|\gamma||\tau|}{4+8 \alpha} \\ 4|h(\mu)| & ;|h(\mu)| \geq \frac{|\gamma||\tau|}{4+8 \alpha}\end{cases}
$$

where

$$
h(\mu)=\frac{(1-\mu) \gamma^{2} \tau^{2}}{4\left[\gamma \tau(1+2 \alpha)+(1+\alpha)^{2}(1-3 \tau)\right]} .
$$

Corollary 3.3. Let $f(z)=z+\sum_{n=2}^{\infty} a_{n} z^{n}$ be in the class $\mathcal{H} \mathcal{S} \mathcal{L}_{\Sigma}(\gamma, \tilde{p})$. Then

$$
\left|a_{2}\right| \leq \frac{|\gamma||\tau|}{\sqrt{3 \gamma \tau+4(1-3 \tau)}}, \quad\left|a_{3}\right| \leq \frac{4|\gamma||\tau|(1-3 \tau)}{3[3 \gamma \tau+4(1-3 \tau)]}
$$

and

$$
\left|a_{3}-\mu a_{2}^{2}\right| \leq \begin{cases}\frac{|\gamma||\tau|}{3} & ; 0 \leq|h(\mu)| \leq \frac{|\gamma||\tau|}{12} \\ 4|h(\mu)| & ;|h(\mu)| \geq \frac{|\gamma||\tau|}{12}\end{cases}
$$

where

$$
h(\mu)=\frac{(1-\mu) \gamma^{2} \tau^{2}}{4[3 \gamma \tau+4(1-3 \tau)]} .
$$


Corollary 3.4. [10] Let $f(z)=z+\sum_{n=2}^{\infty} a_{n} z^{n}$ be in the class $\mathcal{S} \mathcal{L}_{\Sigma}(\tilde{p})$. Then

$$
\left|a_{2}\right| \leq \frac{|\tau|}{\sqrt{1-2 \tau}}, \quad\left|a_{3}\right| \leq \frac{|\tau|(1-4 \tau)}{2-4 \tau}
$$

and

$$
\left|a_{3}-\mu a_{2}^{2}\right| \leq \begin{cases}\frac{|\tau|}{2} & ; 0 \leq|\mu-1| \leq \frac{1-2 \tau}{2|\tau|} \\ \frac{|1-\mu| \tau^{2}}{1-2 \tau} & ;|\mu-1| \geq \frac{1-2 \tau}{2|\tau|}\end{cases}
$$

Corollary 3.5. 10] Let $f(z)=z+\sum_{n=2}^{\infty} a_{n} z^{n}$ be in the class $\mathcal{K} \mathcal{S} \mathcal{L}_{\Sigma}(\tilde{p})$. Then

$$
\left|a_{2}\right| \leq \frac{|\tau|}{\sqrt{4-10 \tau}}, \quad\left|a_{3}\right| \leq \frac{|\tau|(1-4 \tau)}{6-15 \tau}
$$

and

$$
\left|a_{3}-\mu a_{2}^{2}\right| \leq \begin{cases}\frac{|\tau|}{6} & ; 0 \leq|\mu-1| \leq \frac{2-5 \tau}{3|\tau|} \\ \frac{|1-\mu| \tau^{2}}{4-10 \tau} & ;|\mu-1| \geq \frac{2-5 \tau}{6|\tau|}\end{cases}
$$

Remark 3.6. Results discussed in Corollaries 3.4 and 3.5 are coincide with bounds obtained in [10, Corollary 1, Corollary 2, Corollary 4 and Corollary 5].

\section{REFERENCES}

[1] R. M. Ali, S. K. Lee, V. Ravichandran, S. Supramanian, Coefficient estimates for bi-univalent MaMinda starlike and convex functions, Appl. Math. Lett. 25 (2012), no. 3, 344-351.

[2] Ş. Altınkaya and S. Yalçin, On the (p, q)-Lucas polynomial coefficient bounds of the bi-univalent function class, Boletin de la Sociedad Matematica Mexicana, (2018), 1-9.

[3] S. Bulut, Coefficient estimates for a class of analytic and bi-univalent functions, Novi Sad J. Math. 43 (2013), no. 2, 59-65.

[4] M. Çağlar, H. Orhan and N. Yağmur, Coefficient bounds for new subclasses of bi-univalent functions, Filomat, 27 (2013), no. 7, 1165-1171.

[5] E. Deniz, Certain subclasses of bi-univalent functions satisfying subordinate conditions, J. Classical Anal. 2 (2013), no. 1, 49-60.

[6] J. Dziok, R. K. Raina, J. Sokół, Certain results for a class of convex functions related to a shell-like curve connected with Fibonacci numbers, Comp. Math. Appl., 61 (2011), 2605-2613.

[7] J. Dziok, R. K. Raina, J. Sokól, On $\alpha$-convex functions related to a shell-like curve connected with Fibonacci numbers, Appl. Math. Comp., 218 (2011), 996-1002.

[8] P. L. Duren, Univalent Functions, Grundlehren der Mathematischen Wissenschaften Series, 259, Springer Verlag, New York, 1983.

[9] V. B. Girgaonkar and S. B. Joshi, Coefficient estimates for certain subclass of bi-univalent functions associated with Chebyshev polynomial, Ganita, 68(1) (2018), $79-85$.

[10] H. Ö. Güney, G. Murugusundaramoorthy and J. Sokół, Subclasses of bi-univalent functions related to shell-like curves connected with Fibonacci numbers, Acta Univ. Sapientiae, Math., 10 (2018), no. $1,70-84$.

[11] J. M. Jahangiri, S. G. Hamidi and S. Abd. Halim, Coefficients of bi-univalent functions with positive real part derivatives, Bull. Malays. Math. Sci. Soc. (2) 37 (2014), no. 3, 633-640.

[12] H. Orhan, N. Magesh and V. K. Balaji, Fekete-Szegö problem for certain classes of Ma-Minda biunivalent functions, Afr. Mat. 27 (2016), no. 5-6, 889-897. 
[13] H. Orhan, N. Magesh and V. K. Balaji, Certain classes of bi-univalent functions with bounded boundary variation, Tbilisi Math. J. 10 (2017), no. 4, 17-27.

[14] C. Pommerenke, Univalent Functions, Vandenhoeck \& Ruprecht, Göttingen, 1975.

[15] R. K. Raina, J. Sokól, Fekete-Szegö problem for some starlike functions related to shell-like curves, Math. Slovaca, 66 (2016), 135-140.

[16] J. Sokół, On starlike functions connected with Fibonacci numbers, Folia Scient. Univ. Tech. Resoviensis, 175 (1999), 111-116.

[17] H. M. Srivastava and D. Bansal, Coefficient estimates for a subclass of analytic and bi-univalent functions, J. Egyptian Math. Soc. 23 (2015), no. 2, 242-246.

[18] H. M. Srivastava, S. Bulut, M. Çă̆lar, N. Yağmur, Coefficient estimates for a general subclass of analytic and bi-univalent functions, Filomat, 27 (2013), no. 5, 831-842.

[19] H. M. Srivastava, S. S. Eker and R. M. Ali, Coefficient bounds for a certain class of analytic and bi-univalent functions, Filomat 29 (2015), no. 8, 1839-1845.

[20] H. M. Srivastava, S. Gaboury and F. Ghanim, Coefficient estimates for some subclasses of $M$-fold symmetric bi-univalent functions, Acta Univ. Apulensis Math. Inform. 41 (2015), 153-164.

[21] H. M. Srivastava, S. Gaboury and F. Ghanim, Initial coefficient estimates for some subclasses of $m$ fold symmetric bi-univalent functions, Acta Math. Sci. Ser. B Engl. Ed. 36 (2016), no. 3, 863-871.

[22] H. M.Srivastava, N. Magesh and J. Yamini, Initial coefficient estimates for bi- $\lambda-$ convex and bi- $\mu-$ starlike functions connected with arithmetic and geometric means, Electronic J. Math. Anal. Appl. 2 (2014), no. 2, $152-162$.

[23] H. M. Srivastava, A. K. Mishra and P. Gochhayat, Certain subclasses of analytic and bi-univalent functions, Appl. Math. Lett. 23 (2010), no. 10, 1188-1192.

[24] H. M. Srivastava, G. Murugusundaramoorthy and N. Magesh, Certain subclasses of bi-univalent functions associated with the Hohlov operator, Global J. Math. Anal. 1 (2013), no. 2, 67-73.

[25] H. Tang, G-T Deng and S-H Li, Coefficient estimates for new subclasses of Ma-Minda bi-univalent functions, J. Ineq. Appl. 317 (2013), 1-10.

[26] P. Zaprawa, On the Fekete-Szegö problem for classes of bi-univalent functions, Bull. Belg. Math. Soc. Simon Stevin 21 (2014), no. 1, 169-178.

Post-Graduate and Research Department of Mathematics,, Government Arts College FOR Men,, Krishnagiri 635001, TAmilnadu, IndiA. e-mail: nmagi_2000@yahoo.co.in

Department of Mathematics, L.N. Govt College, Ponneri, Chennai, Tamilnadu, India. E-MAIL: balajilsp@yahoo.co.in

Faculty of EngineEring and TeChnology, SRM University, Kattankulathur-603203, TAMILNADU, INDIA. E-MAIL: shreelekha07@yahoo.com 